\title{
HEAT KERNEL ESTIMATES AND THE GREEN FUNCTIONS ON MULTIPLIER HERMITIAN MANIFOLDS
}

\author{
TOSHIKI MABUCHI
}

(Received June 7, 2000)

\begin{abstract}
Using a standard technique of $\mathrm{Li}$ and Yau, we study heat kernel estimates for a special type of compact conformally Kähler manifold, called a multiplier Hermitian manifold of type $\sigma$, which we derive from a Hamiltonian holomorphic vector field on the manifold. In particular, we obtain a lower bound estimate for the Green function averaged by the associated group action. For a fixed $\sigma$, such an estimate is known to play a crucial role in the proof of the uniqueness, modulo a group action, of Einstein multiplier Hermitian structures on a given Fano manifold.
\end{abstract}

1. Introduction. For an $n$-dimensional compact connected Kähler manifold $(M, \omega)$ and a real-valued smooth function $\psi \in C^{\infty}(M)_{R}$ on $M$, we consider the Hermitian form

$$
h_{\psi}:=e^{-\psi / n} \omega,
$$

which naturally defines a conformally Kähler metric on $M$. $\operatorname{Put}^{v_{0} l_{\psi}}(Z):=\int_{Z} h_{\psi}^{n}$ for each measurable subset $Z$ of $M$. The $\operatorname{Ricci}$ form $\operatorname{Ric}\left(h_{\psi}\right)$ associated to $h_{\psi}$ is defined by

$$
\operatorname{Ric}\left(h_{\psi}\right)=\operatorname{Ric}(\omega)+\sqrt{-1} \partial \bar{\partial} \psi, \quad \psi \in C^{\infty}(M)_{\boldsymbol{R}} .
$$

For a system $\left(z^{1}, z^{2}, \ldots, z^{n}\right)$ of holomorphic local coordinates on $M$, the Kähler form $\omega$ is locally written as

$$
\omega=\sqrt{-1} \sum_{\alpha, \beta} g_{\alpha \bar{\beta}} d z^{\alpha} \wedge d z^{\bar{\beta}}
$$

Let $\square_{\omega}$ denote the Laplacian $\sum_{\alpha, \beta} g^{\bar{\beta} \alpha} \partial^{2} / \partial z^{\alpha} \partial z^{\bar{\beta}}$ of the Kähler metric $\omega$. We now define an operator $D_{\psi}$ acting on $C^{\infty}(M)_{C}$ by (cf. [L2], [K1], [Mat])

$$
D_{\psi}:=\square_{\omega}-\sum_{\alpha, \beta} g^{\bar{\beta} \alpha} \frac{\partial \psi}{\partial z^{\alpha}} \frac{\partial}{\partial z^{\bar{\beta}}}, \quad \psi \in C^{\infty}(M)_{\boldsymbol{R}} .
$$

For brevity, we put $P:=\left(D_{\psi}+\bar{D}_{\psi}\right) / 2$, which is the real part of $D_{\psi}$. Let $H=H(x, y, t)$ denote the fundamental solution on $M \times M \times[0, \infty)$ of the equation

$$
(P-\partial / \partial t) v(x, y)=0 .
$$


The Green function $G(x, y) \in C^{\infty}(M \times M \backslash \Delta M)_{R}$ for the operator $P$ is defined outside the diagonal subset $\Delta M$ of $M \times M$, and is characterized by the properties

$$
\left\{\begin{array}{l}
\int_{M} G(x, y) h_{\psi}^{n}(y)=0 \\
f(x)=\operatorname{vol}_{\psi}(M)^{-1} \int_{M} f(y) h_{\psi}^{n}(y)-\int_{M} G(x, y)(P f)(y) h_{\psi}^{n}(y),
\end{array}\right.
$$

where $x \in M$ and $f \in C^{\infty}(M)_{R}$ are arbitrary. From now on, we fix once for all a holomorphic vector field $X \neq 0$ on the Kähler manifold $(M, \omega)$. Furthermore, $X$ is assumed to be Hamiltonian $^{\dagger}$ with respect to $\omega$, i.e., $X$ is expressible as

$$
\operatorname{grad}_{\omega}^{C} u_{\omega}:=\frac{1}{\sqrt{-1}} \sum_{\alpha, \beta} g^{\bar{\beta} \alpha} \frac{\partial u_{\omega}}{\partial z^{\bar{\beta}}} \frac{\partial}{\partial z^{\alpha}}
$$

on $M$ for some $u_{\omega} \in C^{\infty}(M)_{R}$, where $u_{\omega}$ is normalized by $\int_{M} u_{\omega} \omega^{n}=0$. Then the image $I_{X}$ of the function $u_{\omega}$ on $M$ is a closed interval $\left[l_{0}, l_{1}\right]$, where $l_{0}:=\min _{M} u_{\omega}$ and $l_{1}:=$ $\max _{M} u_{\omega}$. Identify $H^{0}(M, \mathcal{O}(T M))$ with the Lie algebra of $\operatorname{Aut}(M)$, where $\operatorname{Aut}(M)$ is the group of all holomorphic automorphisms of $M$. Let $Q$ be the compact torus obtained as the closure in $\operatorname{Aut}(M)$ of the real one-parameter subgroup $\left\{\exp \left(t X_{\boldsymbol{R}}\right) ; t \in \boldsymbol{R}\right\}$ of $\operatorname{Aut}(M)$, where $X_{R}:=X+\bar{X}$ denotes the real vector field on $M$ associated to the holomorphic vector field $X$. Taking the averages of $H$ and $G$ by the $Q$-action, we define $\tilde{H} \in C^{\infty}(M \times M \times(0, \infty))_{\boldsymbol{R}}$ and $\tilde{G} \in C^{\infty}(M \times M \backslash \Delta M)_{R}$ by

$$
\left\{\begin{array}{l}
\tilde{H}(x, y, t):=\int_{Q} H(q \cdot x, y, t) d \mu(q)=\int_{Q} H(x, q \cdot y, t) d \mu(q), \\
\tilde{G}(x, y):=\int_{Q} G(q \cdot x, y) d \mu(q)=\int_{Q} G(x, q \cdot y) d \mu(q),
\end{array}\right.
$$

where $d \mu=d \mu(q)$ denotes the Haar measure for the compact torus $Q$ of total volume 1. For $h_{\psi}$ above, let $0<k \in \boldsymbol{R}$, and let $\sigma=\sigma(s), s \in I_{X}$, be a nonconstant function in $C^{\infty}\left(I_{X}\right)_{\boldsymbol{R}}$ satisfying either of the following conditions:

$$
\begin{array}{ll}
\ddot{\sigma}(s)>0 & \text { on } I_{X}, \\
\ddot{\sigma}(s) \geq 0 & \text { on } I_{X} \quad \text { and } \quad \max _{M} \square_{\omega}(-\psi) \leq k,
\end{array}
$$

where $\dot{\sigma}:=(\partial / \partial s) \sigma$ and $\ddot{\sigma}:=\left(\partial^{2} / \partial s^{2}\right) \sigma$. Then $\left(M, h_{\psi}\right)$ is called a multiplier Hermitian manifold of type $\sigma$, if for some $\sigma$ as above, $\psi$ is written as

$$
\psi=\sigma\left(u_{\omega}\right)
$$

on $M$. Let Diam(M, $\omega)$ be the diameter of the Kähler manifold $(M, \omega)$. For every $x, y \in M$, we consider the distance $r(x, y)$ of $x$ and $y$ on the Kähler manifold $(M, \omega)$. For any $x \in M$

\footnotetext{
$\dagger$ If $X$ is Hamiltonian with respect to $\omega$, then $L_{X_{\boldsymbol{R}}} \omega=0$. By abuse of terminology, if $X$ is Hamiltonian with respect to some Kähler form on $M$, we simply say that $X$ is Hamiltonian. Recall that if $X$ is Hamiltonian, then $X$ is Hamiltonian with respect to any Kähler form $\omega$ on $M$ satisfying $L_{X_{\boldsymbol{R}}} \omega=0$.
} 
and a real number $R>0$, let $B_{x}(R)$ denote the closed ball $\{z \in M ; r(x, z) \leq R\}$ in $M$. We further put

$$
\tilde{B}_{x}(R):=\bigcap_{q \in Q} q\left(B_{x}(R)\right),
$$

which is maximal among the subsets of $B_{x}(R)$ preserved by the $Q$-action on $M$. For any nonnegative real number $t \geq 0$, we put

$$
\left\{\begin{array}{l}
\underline{t}:=\min \left\{t, \operatorname{Diam}(M, \omega)^{2}\right\}, \\
\{r(x, y)-\sqrt{t}\}_{+}:=\max \{r(x, y)-\sqrt{t}, 0\} .
\end{array}\right.
$$

The main purpose of this paper is, given a multiplier Hermitian manifold $\left(M, h_{\psi}\right)$ of type $\sigma$, to establish an upper bound ${ }^{\dagger}$ of the average heat kernel $\tilde{H}$ and also a lower bound of the average Green function $\tilde{G}$ as follows:

THEOREM A. Let $\left(M, h_{\psi}\right)$ be a multiplier Hermitian manifold of type $\sigma$. Suppose that the Ricci form $\operatorname{Ric}\left(h_{\psi / 2}\right)$ is positive semidefinite everywhere on $M$. Then for every $\varepsilon \in \boldsymbol{R}$ with $0<\varepsilon \leq 1$, we have:

(a) If (1.5) is the case, then for some positive real constants $C_{1}=C_{1}(\sigma, n)$ depending only on the pair $(\sigma, n)$, we have the following estimate for all $0<t \in \boldsymbol{R}$ and all $x, y \in M$ :

$$
\left\{\begin{array}{l}
\operatorname{vol}_{\psi}\left(\tilde{B}_{x}(\sqrt{t})\right)^{1 / 2} \operatorname{vol}_{\psi}\left(\tilde{B}_{y}(\sqrt{t})\right)^{1 / 2} \tilde{H}(x, y, t) \\
\leq(1+\sqrt{\varepsilon})^{2 n / C_{1}} \exp \left\{-\frac{\{r(x, y)-\sqrt{t}\}_{+}^{2}}{2 t(1+2 \varepsilon)}+\frac{7}{3} \cdot \frac{t}{t \varepsilon}+\frac{4 k \varepsilon t}{3 C_{1}}\right\}
\end{array}\right.
$$

(b) If (1.4) is the case, then for some positive real constant $C_{0}=C_{0}(\sigma, n)$ depending only on the pair $(\sigma, n)$, we have the following estimate for all $0<t \in \boldsymbol{R}$ and all $x, y \in M$ :

$$
\left\{\begin{array}{l}
\operatorname{vol}_{\psi}\left(\tilde{B}_{x}(\sqrt{t})\right)^{1 / 2} \operatorname{vol}_{\psi}\left(\tilde{B}_{y}(\sqrt{t})\right)^{1 / 2} \tilde{H}(x, y, t) \\
\leq(1+\sqrt{\varepsilon})^{2 / C_{0}} \exp \left\{-\frac{\{r(x, y)-\sqrt{t}\}_{+}^{2}}{2 t(1+2 \varepsilon)}+\frac{7}{3} \cdot \frac{t}{t \varepsilon}\right\} .
\end{array}\right.
$$

THEOREM B. Let $\left(M, h_{\psi}\right)$ be a multiplier Hermitian manifold of type $\sigma$, and let $v>0$ be a positive real number. Suppose that $\operatorname{Ric}\left(h_{\psi / 2}\right) \geq v \omega$, i.e., $\operatorname{Ric}\left(h_{\psi / 2}\right)-v \omega$ is positive semidefinite on $M$. Then

(a) If (1.5) is the case, then for some positive real constants $k_{1}=k_{1}(\sigma, n), k_{2}=$ $k_{2}(\sigma, n)$ and $k_{3}=k_{3}(\sigma, n)$ depending only on $(\sigma, n)$, we have

$$
\tilde{G}(x, y) \geq-\left\{v \operatorname{vol}_{\psi}(M)\right\}^{-1}\left(k_{1}+k_{2} e^{k_{3} k / v}\right) \text { for all } x, y \in M \text { with } x \neq y .
$$

(b) If (1.4) is the case, then for some positive real constant $k_{0}=k_{0}(\sigma, n)$ depending only on the pair $(\sigma, n)$, we have

$$
\tilde{G}(x, y) \geq-\left\{v \operatorname{vol}_{\psi}(M)\right\}^{-1} k_{0} \text { for all } x, y \in M \text { with } x \neq y .
$$

\footnotetext{
$\dagger$ See [S1] for some similar result in Riemannian cases. His result is not applicable to ours, because in our Kähler cases, the lower boundedness of his symmetric tensor $R_{w}$ is definitely stronger than the lower boundedness of our Ricci form $\operatorname{Ric}\left(h_{\psi / 2}\right)$, where $\psi=-2 \log w$.
} 
In actual applications of the theorems ${ }^{\dagger}$, the following Theorem $\mathrm{C}$ is sometimes useful (see [M1]). Let $X \neq 0$ be a holomorphic vector field on $M$ which is Hamiltonian in the sense of a footnote in this introduction. For a fixed $\sigma$, assuming (1.6), we consider the multiplier Hermitian manifolds

$$
\left(M, h_{\sigma\left(u_{\omega}\right)}\right), \quad \omega \in \mathcal{K}_{X},
$$

of type $\sigma$, where $\mathcal{K}_{X}$ denotes the set of all Kähler forms on $M$ in the class $2 \pi c_{1}(M)_{\boldsymbol{R}}$ such that $L_{X_{R}} \omega=0$. Fix an element $\omega_{0}$ in $\mathcal{K}_{X}$. Let $\mathcal{F}_{\sigma}$ be the set of all $\omega \in \mathcal{K}_{X}$ such that

$$
\operatorname{Ric}\left(h_{\sigma\left(u_{\omega}\right)}\right)=t \omega+(1-t) \omega_{0}
$$

for some $t \in[0,1]$. We here observe that (1.9) is an analogue of Aubin's equation (cf. [A1]). This family $\mathcal{F}_{\sigma}$ is shown to have a very nice property as follows:

THEOREM C. Assume that $\dot{\sigma} \leq 0 \leq \ddot{\sigma}$ on $I_{X}$. Then for some real constant $k_{\sigma}>0$, the inequality $\max _{M} \square_{\omega}\left\{-\sigma\left(u_{\omega}\right)\right\} \leq k_{\sigma}$ holds uniformly for all $\omega \in \mathcal{F}_{\sigma}$.

Parts of this work were done during my stay at the International Centre for Mathematical Sciences (ICMS), Edinburgh in 1997. I thank especially Professor Michael Singer who invited me to give lectures in ICMS on various subjects related to Kähler-Einstein metrics.

2. The first eigenvalue of the operator $\boldsymbol{D}_{\psi}$. Let $\omega$ be as in the introduction. For each $\psi \in C^{\infty}(M)_{R}$, we consider the operator $D_{\psi}$. For complex-valued functions $v_{1}, v_{2} \in$ $C^{\infty}(M)_{C}$ on $M$, we put (cf. [L2], [K1], [Mat], [F1])

$$
\left\langle\left\langle v_{1}, v_{2}\right\rangle\right\rangle:=\int_{M} v_{1} \bar{v}_{2} h_{\psi}^{n}=\int_{M} v_{1} \bar{v}_{2} e^{-\psi} \omega^{n} .
$$

In the arguments in [F1; p. 41], we replace the function $F$ by $\psi$. Then $D_{\psi}$ is easily shown to be self-adjoint with respect to the above Hermitian inner product as follows:

$$
\begin{aligned}
& \left\langle\left\langle v_{1}, D_{\psi} v_{2}\right\rangle\right\rangle=\int_{M} v_{1}\left\{\overline{\square_{\omega} v_{2}}-\left(\bar{\partial} \psi, \bar{\partial} v_{2}\right)_{\omega}\right\} e^{-\psi} \omega^{n} \\
& =\int_{M}\left\{-\left(\bar{\partial}\left(v_{1} e^{-\psi}\right), \bar{\partial} v_{2}\right)_{\omega}-v_{1}\left(\bar{\partial} \psi, \bar{\partial} v_{2}\right)_{\omega} e^{-\psi}\right\} \omega^{n}=-\int_{M}\left(\bar{\partial} v_{1}, \bar{\partial} v_{2}\right)_{\omega} e^{-\psi} \omega^{n}, \\
& \left\langle\left\langle D_{\psi} v_{1}, v_{2}\right\rangle\right\rangle=\int_{M}\left\{\square_{\omega} v_{1}-\left(\bar{\partial} v_{1}, \bar{\partial} \psi\right)_{\omega}\right\} \bar{v}_{2} e^{-\psi} \omega^{n} \\
& =\int_{M}\left\{-\left(\bar{\partial} v_{1}, \bar{\partial}\left(e^{-\psi} v_{2}\right)\right)_{\omega}-\left(\bar{\partial} v_{1}, \bar{\partial} \psi\right)_{\omega} \bar{v}_{2} e^{-\psi}\right\} \omega^{n}=-\int_{M}\left(\bar{\partial} v_{1}, \bar{\partial} v_{2}\right)_{\omega} e^{-\psi} \omega^{n} .
\end{aligned}
$$

Moreover, by $\left\langle\left\langle v, D_{\psi} v\right\rangle\right\rangle=-\int_{M}(\bar{\partial} v, \bar{\partial} v)_{\omega} e^{-\psi} \omega^{n} \leq 0$, all eigenvalues of $-D_{\psi}$ are nonnegative real numbers. Therefore, the real part $P$ of $D_{\psi}$ satisfies

(2.1) $-P$ is a real selfadjoint elliptic operator only with nonnegative eigenvalues.

${ }^{\dagger}$ Related to the theorems, the study of multiplier Hermitian analogues of spectral convergence (cf. [KK]) would also be an interesting topic. 
Let $\lambda_{1}=\lambda_{1}(\omega, \psi)>0$ be the first positive eigenvalue of the operator $-D_{\psi}$, and assume for some positive real number $v$

$$
\operatorname{Ric}\left(h_{\psi}\right) \geq v \omega \quad \text { on } M .
$$

Then $c_{1}(M)>0$, and by the Kodaira vanishing theorem, we have $0=h^{0,1}(M)=h^{1,0}(M)$.

Let $\mathfrak{g}:=H^{0}(M, \mathcal{O}(T M))$ be the space of all holomorphic vector fields on $M$. Then we have a $\boldsymbol{C}$-linear isomorphism of complex vector spaces

$$
\mathfrak{g}^{\omega} \cong \mathfrak{g}, \quad u \leftrightarrow \operatorname{grad}_{\omega}^{C} u,
$$

where $\mathfrak{g}^{\omega}$ denotes the space of all $v \in C^{\infty}(M)_{C}$ satisfying $\operatorname{grad}_{\omega}^{C} v \in \mathfrak{g}$ and $\int_{M} v h_{\psi}^{n}=0$. Then the following more or less standard fact is a slight generalization of [L1] and [F1; Theorem 2.4.3] (see also [B1; p. 140], [DS], [S2], [TZ]):

PROPOSITION 2.3. Assuming (2.2), we have

(1) $\lambda_{1}(\omega, \psi) \geq v$.

(2) If $\lambda_{1}(\omega, \psi)=v$, then $\left\{v \in C^{\infty}(M)_{C} ; D_{\psi} v=-\lambda_{1}(\omega, \psi) v\right\}$ is a subspace of $\mathfrak{g}^{\omega}$.

Proof. The proof is similar to [F1; p. 41-42] and proceeds as follows: Let $v$ be an eigenfunction for $-D_{\psi}$ with eigenvalue $\lambda_{1}=\lambda_{1}(\omega, \psi)$. Then $v$ is not a constant function. Put $Y_{v}:=\operatorname{grad}_{\omega}^{C} v \neq 0$. We write $\operatorname{Ric}(\omega)=\sqrt{-1} \sum_{\alpha, \beta} R_{\alpha \bar{\beta}} d z^{\alpha} \wedge d z^{\bar{\beta}}$ and $R_{\gamma}^{\alpha}:=\sum_{\beta} R_{\gamma \bar{\beta}} g^{\bar{\beta} \alpha}$ by using holomorphic local coordinates. It then follows that

$$
\begin{aligned}
& \lambda_{1} \int_{M}\left(Y_{v}, Y_{v}\right)_{\omega} e^{-\psi} \omega^{n}=\lambda_{1} \int_{M}(\bar{\partial} v, \bar{\partial} v)_{\omega} e^{-\psi} \omega^{n}=\lambda_{1} \int_{M} \sum g^{\bar{\beta} \alpha} \frac{\partial v}{\partial z^{\bar{\beta}}} \frac{\partial \bar{v}}{\partial z^{\alpha}} e^{-\psi} \omega^{n} \\
& =\int_{M} \sum g^{\bar{\beta} \alpha} \frac{\partial\left(-D_{\psi} v\right)}{\partial z^{\bar{\beta}}} \frac{\partial \bar{v}}{\partial z^{\alpha}} e^{-\psi} \omega^{n}=-\int_{M}\left\{\sum \nabla^{\alpha}\left(\nabla_{\gamma} \nabla^{\gamma} v-\nabla^{\gamma} v \nabla_{\gamma} \psi\right) \nabla_{\alpha} \bar{v}\right\} e^{-\psi} \omega^{n} \\
& =-\int_{M} \sum_{\alpha, \gamma}\left\{\left(\nabla_{\gamma} \nabla^{\alpha} \nabla^{\gamma} v\right) \nabla_{\alpha} \bar{v}-R_{\gamma}{ }^{\alpha} \nabla^{\gamma} v \nabla_{\alpha} \bar{v}-\nabla^{\alpha}\left(\nabla^{\gamma} v \nabla_{\gamma} \psi\right) \nabla_{\alpha} \bar{v}\right\} e^{-\psi} \omega^{n} \\
& =\int_{M} \sum_{\alpha, \gamma}\left\{\left(\nabla^{\alpha} \nabla^{\gamma} v\right)\left(\nabla_{\gamma} \nabla_{\alpha} \bar{v}\right)+R_{\gamma}{ }^{\alpha} \nabla^{\gamma} v \nabla_{\alpha} \bar{v}+\left(\nabla^{\gamma} v\right)\left(\nabla^{\alpha} \nabla_{\gamma} \psi\right)\left(\nabla_{\alpha} \bar{v}\right)\right\} e^{-\psi} \omega^{n},
\end{aligned}
$$

where the last equality is obtained by using integration by parts. We observe that by (2.2), $\operatorname{Ric}\left(h_{\psi}\right) \geq v \omega$. Hence, together with (1.1), we obtain

$$
\begin{gathered}
\lambda_{1} \int_{M}\left(Y_{v}, Y_{v}\right)_{\omega} e^{-\psi} \omega^{n}=\int_{M}\left\{\left(\bar{\partial} Y_{u}, \bar{\partial} Y_{u}\right)_{\omega}+\operatorname{Ric}\left(h_{\omega}^{\psi}\right)\left(Y_{v} \wedge \bar{Y}_{v} / \sqrt{-1}\right)\right\} e^{-\psi} \omega^{n} \\
\geq \int_{M}\left\{\left(\bar{\partial} Y_{v}, \bar{\partial} Y_{v}\right)_{\omega}+v\left(Y_{v}, Y_{v}\right)_{\omega}\right\} e^{-\psi} \omega^{n} \geq v \int_{M}\left(Y_{v}, Y_{v}\right)_{\omega} e^{-\psi} \omega^{n} .
\end{gathered}
$$

Therefore, the inequality $\lambda_{1} \geq v$ in (1) above holds. To see (2), we assume that $\lambda_{1}=v$. Then by (2.4), we see that $\bar{\partial} Y_{v}=0$, i.e., $Y_{v} \in \mathfrak{g}$. This together with the equalities $\lambda_{1}\langle\langle v, 1\rangle\rangle=$ $\left\langle\left\langle D_{\psi} v, 1\right\rangle\right\rangle=\left\langle\left\langle v, D_{\psi} 1\right\rangle\right\rangle=0$ implies $v \in \mathfrak{g}^{\omega}$, as required.

Let $l \geq 0$ be an arbitrary nonnegative real number. we here study the first positive eigenvalue $\lambda_{1}(\omega, l \psi)>0$ of the operator $-D_{l \psi}$ on $C^{\infty}(M)$, where we assume that (2.2) 
holds for some positive real number $v$. Put

$$
c:=\max _{M}|\psi|
$$

Let $\mathcal{S}$ denote the space of all nonconstant functions in $C^{\infty}(M)_{C}$. We then define a functional $\Phi_{l}: \mathcal{S} \rightarrow \boldsymbol{R}$ by

$$
\Phi_{l}(f):=\frac{\int_{M}(\bar{\partial} f, \bar{\partial} f)_{\omega} e^{-l \psi} \omega^{n}}{\min _{b \in \boldsymbol{C}} \int_{M}|f+b|^{2} e^{-l \psi} \omega^{n}}, \quad f \in \mathcal{S} .
$$

Note that $\lambda_{1}(\omega, l \psi)=\min _{f \in \mathcal{S}} \Phi_{l}(f)$. In view of (1) of Proposition 2.3, for all $f \in \mathcal{S}$, we have the following :

$$
\begin{aligned}
\Phi_{l}(f) & \geq \frac{\int_{M}(\bar{\partial} f, \bar{\partial} f)_{\omega} \exp (-|l-1| c) e^{-\psi} \omega^{n}}{\min _{b \in C} \int_{M}|f+b|^{2} \exp (|l-1| c) e^{-\psi} \omega^{n}} \\
& =\exp (-2|l-1| c) \frac{\int_{M}(\bar{\partial} f, \bar{\partial} f)_{\omega} e^{-\psi} \omega^{n}}{\min _{b \in C} \int_{M}|f+b|^{2} e^{-\psi} \omega^{n}} \\
& \geq \exp (-2|l-1| c) \lambda_{1}(\omega, \psi) \geq v \exp (-2|l-1| c) .
\end{aligned}
$$

Let $f$ run through $\mathcal{S}$. Taking the minimum, we obtain

PROPOSITION 2.6. Assuming (2.2), we have $\lambda_{1}(\omega, l \psi) \geq v \exp (-2|l-1| c)$ for every real number $l$, where $c$ is as in (2.5).

3. Constants $C_{0}, C_{1}$ and positive solutions of the heat equation (1.3). In this section, after defining explicitly the constants $C_{0}$ and $C_{1}$ of Theorem A, we study basic properties of positive smooth solutions of (1.3). As in Theorem A, we assume that

$$
\psi=\sigma\left(u_{\omega}\right) \quad \text { and } \quad \operatorname{Ric}\left(h_{\psi / 2}\right) \geq 0
$$

on $M$ for $\sigma$ as in the introduction, so that $\sigma$ is a nonconstant function in $C^{\infty}\left(I_{X}\right)_{\boldsymbol{R}}$ satisfying either (1.4) or (1.5). In particular, $\ddot{\sigma} \geq 0$ on $I_{X}$. Put

$$
c:=\max _{s \in I_{X}}|\sigma(s)|, \quad \underline{c}:=\min _{s \in I_{X}} \sigma(s), \quad \bar{c}:=\max _{s \in I_{X}} \sigma(s),
$$

and the first identity is compatible with (2.5) in Section 2. Since $X$ is Hamiltonian with respect to $\omega$, we have $L_{X_{\boldsymbol{R}}} \omega=0$ for $X_{\boldsymbol{R}}:=X+\bar{X}$ as in the introduction. By (1.2), we have

$$
\bar{D}_{\psi}-D_{\psi}=-\sqrt{-1} \dot{\sigma}(u) X_{\boldsymbol{R}} .
$$

For the space $C^{\infty}(M \times(0, \infty))_{R}$ of all real-valued $C^{\infty}$ functions $f=f(x, t)$ on $M \times$ $(0, \infty)=\{(x, t) \in M \times \boldsymbol{R} ; t>0\}$, we define a subspace $\Sigma$ by

$$
\Sigma:=\left\{f \in C^{\infty}(M \times(0, \infty))_{\boldsymbol{R}} ; X_{\boldsymbol{R}} f=0\right\} .
$$


Then $D_{\psi} f=\bar{D}_{\psi} f$ for all $f \in \Sigma$. If $\ddot{\sigma}>0$ on $I_{X}$, i.e., if (1.4) is the case, then we can define positive real constants $C$ and $C_{0}=C_{0}(\sigma, n)$ depending only on the pair $(\sigma, n)$ by

$$
\left\{\begin{array}{l}
C:=\left\{\max _{s \in I_{X}} \ddot{\sigma}(s)^{-1} \dot{\sigma}(s)^{2}\right\}^{-1}, \\
C_{0}:=n^{-1}(C+1)^{-1} C .
\end{array}\right.
$$

In general, even if the function $\ddot{\sigma}=\ddot{\sigma}(s)$ has zeroes on $I_{X}$, we define a monotone-decreasing real-valued function $\xi:[\underline{c}, \bar{c}] \rightarrow \boldsymbol{R}$ by

$$
\xi(s):=\exp \int_{\underline{c}}^{s} \frac{1}{b_{0} e^{-y}-1} d y, \quad \underline{c} \leq s \leq \bar{c},
$$

where $b_{0}:=e^{\bar{c}}(1+n)$. Then $\xi=\xi(s)$ takes its absolute minimum 1 at $s=\underline{c}$, and satisfies the differential equation

$$
\frac{\ddot{\xi}(s)}{\xi(s)}-\frac{\dot{\xi}(s)}{\xi(s)}=2\left\{\frac{\dot{\xi}(s)}{\xi(s)}\right\}^{2}
$$

for all $s$. Define a positive real constant $C_{1}=C_{1}(\sigma, n)$ depending only on $(\sigma, n)$ by

$$
C_{1}:=\min _{s \in[\underline{c}, \bar{c}]} \xi(s)^{-1}\left\{1-\left(1-\xi(s)^{-1} \dot{\xi}(s) n\right)^{2}\right\} .
$$

In the arguments below, standard techniques in [LY] (see also [D1]) are employed. For each function in $\Sigma$, the operator $d:=\partial+\bar{\partial}$ is taken only on the first factor $M$ of $M \times[0, \infty)$. Note that the following inequalities holds for all $s \in[\underline{c}, \bar{c}]$ :

$$
\left\{\begin{array}{l}
0<C_{1} \leq \xi(s)^{-1}\left\{1-\left(1-\xi(s)^{-1} \dot{\xi}(s) n\right)^{2}\right\} \\
0<\xi(s)^{-1} \dot{\xi}(s) \leq 1 / n
\end{array}\right.
$$

Proposition 3.3. Assume that a function $f=f(x, t)$ in $\Sigma$ satisfies $(P-\partial / \partial t) f=$ $-(\bar{\partial} f, \bar{\partial} f)_{\omega}$ everywhere on $M \times(0, \infty)$. Put $f_{t}:=\partial f / \partial t, \hat{F}:=t\left\{(\bar{\partial} f, \bar{\partial} f)_{\omega}-f_{t}\right\}$ and $F:=\xi(\psi) \hat{F}$. Then $F$ satisfies the following inequality on $M \times(0, \infty)$ :

$$
(P-\partial / \partial t) F \geq-(d F, d f)_{\omega}+\frac{\dot{\xi}(\psi)}{\xi(\psi)}\left\{(d \psi, d F)_{\omega}+\left(\square_{\omega} \psi\right) F\right\}-\frac{F}{t}+C_{1} \frac{F^{2}}{n t} .
$$

In the case where the inequality $\ddot{\sigma}>0$ holds on $I_{X}$, the function $\hat{F}$ satisfies

$$
(P-\partial / \partial t) \hat{F} \geq-(d \hat{F}, d f)_{\omega}-\frac{\hat{F}}{t}+C_{0} \frac{\hat{F}^{2}}{t} .
$$

Proof. In a neighbourhood of each point $x$ of $M$, choose a system $\left(z^{1}, z^{2}, \ldots, z^{n}\right)$ of holomorphic local coordinates as in the introduction, where we may assume that $g_{\alpha \bar{\beta}}(x)=$ $\delta_{\alpha \beta}$ and $\left(d g_{\alpha \bar{\beta}}\right)(x)=0$ for all $\alpha$ and $\beta$. We write $\hat{F}=t\left\{\sum_{\alpha}\left(\nabla^{\alpha} f\right)\left(\nabla_{\alpha} f\right)-f_{t}\right\}$. Then

$$
\begin{aligned}
D_{\psi} \hat{F} & -t\left\{\sum_{\alpha}\left(D_{\psi} \nabla^{\alpha} f\right) \nabla_{\alpha} f+\sum_{\alpha}\left(\nabla^{\alpha} f\right)\left(D_{\psi} \nabla_{\alpha} f\right)-D_{\psi} f_{t}\right\} \\
=t & \sum_{\alpha, \gamma}\left\{\left(\nabla^{\gamma} \nabla^{\alpha} f\right)\left(\nabla_{\gamma} \nabla_{\alpha} f\right)+\left(\nabla_{\gamma} \nabla^{\alpha} f\right)\left(\nabla^{\gamma} \nabla_{\alpha} f\right)\right\} \geq n^{-1} t\left(\square_{\omega} f\right)^{2} .
\end{aligned}
$$


Before (3.3.5), all inequalities below are considered only at the point $x$. For (3.3.5) and thereafter, inequalities in this proof are valid also on the whole $M$, because $x$ can be chosen as an arbitrary point of $M$. Now for simplicity, put $Y_{f}:=\operatorname{grad}_{\omega}^{C} f$. Since $\operatorname{Ric}\left(h_{\psi / 2}\right)$ is positive semidefinite, we have the inequality $\operatorname{Ric}\left(h_{\psi / 2}\right)\left(Y_{f} \wedge \bar{Y}_{f} / \sqrt{-1}\right) \geq 0$. Hence, as in the proof of Proposition 2.3,

$$
\begin{aligned}
& \left(\bar{\partial}\left(D_{\psi} f\right), \bar{\partial} f\right)_{\omega}=\sum_{\alpha, \gamma}\left\{\nabla^{\alpha}\left(\nabla_{\gamma} \nabla^{\gamma} f-\nabla^{\gamma} f \nabla_{\gamma} \psi\right)\right\} \nabla_{\alpha} f \\
& =\sum_{\alpha, \gamma}\left\{\nabla_{\gamma} \nabla^{\gamma} \nabla^{\alpha} f-R_{\gamma}{ }^{\alpha} \nabla^{\gamma} f-\left(\nabla^{\gamma} f\right)\left(\nabla^{\alpha} \nabla_{\gamma} \psi\right)-\left(\nabla^{\gamma} \nabla^{\alpha} f\right) \nabla_{\gamma} \psi\right\} \nabla_{\alpha} f \\
& =\sum_{\alpha}\left(\square_{\omega} \nabla^{\alpha} f\right) \nabla_{\alpha} f-\left\{\operatorname{Ric}\left(h_{\psi}\right)\left(Y_{f} \wedge \bar{Y}_{f} / \sqrt{-1}\right)-\sum_{\alpha, \gamma}\left(\nabla^{\gamma} \nabla^{\alpha} f\right)\left(\nabla_{\gamma} \psi\right)\left(\nabla_{\alpha} f\right)\right. \\
& =\sum_{\alpha}\left(D_{\psi} \nabla^{\alpha} f\right) \nabla_{\alpha} f-\left\{\operatorname{Ric}\left(h_{\psi / 2}\right)+2^{-1} \sqrt{-1} \partial \bar{\partial} \psi\right\}\left(Y_{f} \wedge \bar{Y}_{f} / \sqrt{-1}\right) \\
& \leq \sum_{\alpha}\left(D_{\psi} \nabla^{\alpha} f\right) \nabla_{\alpha} f-2^{-1}(\partial \bar{\partial} \psi)\left(Y_{f} \wedge \bar{Y}_{f}\right),
\end{aligned}
$$

where we used the equality $\operatorname{Ric}\left(h_{\psi}\right)=\operatorname{Ric}\left(h_{\psi / 2}\right)+2^{-1} \sqrt{-1} \partial \bar{\partial} \psi$. Since $f \in \Sigma$, we have $\bar{D}_{\psi} f=D_{\psi} f$. Then the same calculation as above yields

$$
\begin{aligned}
& \left(\partial\left(D_{\psi} f\right), \partial f\right)_{\omega}=\left(\bar{\partial} f, \bar{\partial}\left(D_{\psi} f\right)\right)_{\omega}=\sum_{\alpha, \gamma}\left(\nabla^{\alpha} f\right)\left\{\nabla_{\alpha}\left(\nabla^{\gamma} \nabla_{\gamma} f-\nabla_{\gamma} f \nabla^{\gamma} \psi\right)\right\} \\
& =\sum_{\alpha, \gamma}\left(\nabla^{\alpha} f\right)\left\{\nabla^{\gamma} \nabla_{\gamma} \nabla_{\alpha} f-R_{\alpha}{ }^{\gamma} \nabla_{\gamma} f-\left(\nabla_{\gamma} f\right)\left(\nabla_{\alpha} \nabla^{\gamma} \psi\right)-\left(\nabla_{\gamma} \nabla_{\alpha} f\right) \nabla^{\gamma} \psi\right\} \\
& =\sum_{\alpha}\left(\nabla^{\alpha} f\right)\left(\square_{\omega} \nabla_{\alpha} f\right)-\operatorname{Ric}\left(h_{\psi}\right)\left(Y_{f} \wedge \bar{Y}_{f} / \sqrt{-1}\right)-\sum_{\alpha, \gamma}\left(\nabla_{\gamma} \nabla_{\alpha} f\right)\left(\nabla^{\gamma} \psi\right)\left(\nabla^{\alpha} f\right) \\
& \leq \sum_{\alpha}\left(\nabla^{\alpha} f\right)\left(\bar{D}_{\psi} \nabla_{\alpha} f\right)-2^{-1}(\partial \bar{\partial} \psi)\left(Y_{f} \wedge \bar{Y}_{f}\right) .
\end{aligned}
$$

We now sum up these two inequalities obtained just above. Since $\bar{D}_{\psi}=D_{\psi}-\sqrt{-1} \dot{\sigma}\left(u_{\omega}\right) X_{\boldsymbol{R}}$, by setting $B:=\sum_{\alpha}\left(\nabla^{\alpha} f\right)\left(X_{\boldsymbol{R}} \nabla_{\alpha} f\right)$, we obtain

$$
\begin{aligned}
& \left(d\left(D_{\psi} f\right), d f\right)_{\omega} \leq \sum_{\alpha}\left(D_{\psi} \nabla^{\alpha} f\right) \nabla_{\alpha} f+\sum_{\alpha}\left(\nabla^{\alpha} f\right)\left(\bar{D}_{\psi} \nabla_{\alpha} f\right)-(\partial \bar{\partial} \psi)\left(Y_{f} \wedge \bar{Y}_{f}\right) \\
& =\sum_{\alpha}\left(D_{\psi} \nabla^{\alpha} f\right) \nabla_{\alpha} f+\sum_{\alpha}\left(\nabla^{\alpha} f\right)\left(D_{\psi} \nabla_{\alpha} f\right)-\sqrt{-1} \dot{\sigma}(u) B-(\partial \bar{\partial} \psi)\left(Y_{f} \wedge \bar{Y}_{f}\right) .
\end{aligned}
$$


Note that $B=\left(\bar{\partial} f, L_{X_{R}} \bar{\partial} f\right)_{\omega}+\sqrt{-1}\left(\partial \bar{\partial} u_{\omega}\right)\left(Y_{f} \wedge \bar{Y}_{f}\right)=\sqrt{-1}\left(\partial \bar{\partial} u_{\omega}\right)\left(Y_{f} \wedge \bar{Y}_{f}\right)$. Further, by $\psi=\sigma\left(u_{\omega}\right)$, we have $\partial \bar{\partial} \psi=\dot{\sigma}\left(u_{\omega}\right) \partial \bar{\partial} u_{\omega}+\ddot{\sigma}\left(u_{\omega}\right) \partial u_{\omega} \wedge \bar{\partial} u_{\omega}$. Hence by (3.3.4),

$$
\begin{aligned}
\left(d\left(D_{\psi} f\right), d f\right)_{\omega} \leq & \sum_{\alpha}\left(D_{\psi} \nabla^{\alpha} f\right) \nabla_{\alpha} f \\
& +\sum_{\alpha}\left(\nabla^{\alpha} f\right)\left(D_{\psi} \nabla_{\alpha} f\right)-\ddot{\sigma}\left(u_{\omega}\right)\left(\partial u_{\omega} \wedge \bar{\partial} u_{\omega}, \partial f \wedge \bar{\partial} f\right)_{\omega},
\end{aligned}
$$

and together with (3.3.3), we have $D_{\psi} \hat{F} \geq t\left(d\left(D_{\psi} f\right), d f\right)_{\omega}-t D_{\psi} f_{t}+n^{-1} t\left(\square_{\omega} f\right)^{2}+$ $t \ddot{\sigma}(u)\left(\partial u_{\omega} \wedge \bar{\partial} u_{\omega}, \partial f \wedge \bar{\partial} f\right)_{\omega}$. Then, by taking its real part, we obtain

$$
P \hat{F} \geq t(d(P f), d f)_{\omega}-t P f_{t}+n^{-1} t\left(\square_{\omega} f\right)^{2}+t \ddot{\sigma}(u)\left(\partial u_{\omega} \wedge \bar{\partial} u_{\omega}, \partial f \wedge \bar{\partial} f\right)_{\omega} .
$$

By $P f-f_{t}=-(\bar{\partial} f, \bar{\partial} f)_{\omega}$, we have $\hat{F}=t\left\{(\bar{\partial} f, \bar{\partial} f)_{\omega}-f_{t}\right\}=-t P f$ and $(\partial / \partial t) \hat{F}-t^{-1} \hat{F}=$ $-t P f_{t}$. We further obtain $\square_{\omega} f=P f+2^{-1}(d \psi, d f)_{\omega}=-t^{-1} \hat{F}+2^{-1}(d \psi, d f)_{\omega}$. By $\ddot{\sigma}(u) \geq 0$, these together with (3.3.5) yield

$$
\left\{\begin{array}{l}
(P-\partial / \partial t) \hat{F} \geq(P-\partial / \partial t) \hat{F}-t \ddot{\sigma}(u)\left(\partial u_{\omega} \wedge \bar{\partial} u_{\omega}, \partial f \wedge \bar{\partial} f\right)_{\omega} \\
\geq-(d \hat{F}, d f)_{\omega}-t^{-1} \hat{F}+n^{-1} t\left(\square_{\omega} f\right)^{2} \\
=-(d \hat{F}, d f)_{\omega}-t^{-1} \hat{F}+n^{-1} t\left\{t^{-1} \hat{F}-2^{-1}(d \psi, d f)_{\omega}\right\}^{2}
\end{array}\right.
$$

By $F=\xi(\psi) \hat{F}$, we see that $P F=\dot{\xi}(\psi)(d \psi, d \hat{F})_{\omega}+P\{\xi(\psi)\} \hat{F}+\xi(\psi) P \hat{F}$, where

$$
\left\{\begin{aligned}
\dot{\xi}(\psi)(d \psi, d \hat{F})_{\omega} & =\xi(\psi)^{-1} \dot{\xi}(\psi)(d \psi, d F)_{\omega}-2 \xi(\psi)^{-2} \dot{\xi}(\psi)^{2} F(\bar{\partial} \psi, \bar{\partial} \psi)_{\omega}, \\
P\{\xi(\psi)\} & =\{\ddot{\xi}(\psi)-\dot{\xi}(\psi)\}(\bar{\partial} \psi, \bar{\partial} \psi)_{\omega}+\dot{\xi}(\psi) \square_{\omega} \psi \\
\xi(\psi)(d \hat{F}, d f)_{\omega} & =(d F, d f)_{\omega}-\xi(\psi)^{-1} \dot{\xi}(\psi) F(d \psi, d f)_{\omega}
\end{aligned}\right.
$$

Hence, multiplying (3.3.6) by $\xi(\psi)$ and rewriting it as an inequality in $F$, we obtain

$$
\begin{aligned}
(P-\partial / \partial t) F \geq & \left(\frac{\ddot{\xi}(\psi)}{\xi(\psi)}-\frac{\dot{\xi}(\psi)}{\xi(\psi)}-2\left\{\frac{\dot{\xi}(\psi)}{\xi(\psi)}\right\}^{2}\right) F(\bar{\partial} \psi, \bar{\partial} \psi)_{\omega}-(d F, d f)_{\omega} \\
& +\xi(\psi)^{-1} \dot{\xi}(\psi)\left\{(d \psi, d F)_{\omega}+\left(\square_{\omega} \psi\right) F\right\}-t^{-1} F \\
& +n^{-1} t^{-1} F^{2} \xi(\psi)^{-1}\left\{1-\left(1-\xi(\psi)^{-1} \dot{\xi}(\psi) n\right)^{2}\right\} \\
& +n^{-1} t \xi(\psi)\left\{2^{-1}(d \psi, d f)_{\omega}-t^{-1} \xi(\psi)^{-1} F\left(1-\xi(\psi)^{-1} \dot{\xi}(\psi) n\right)\right\}^{2}
\end{aligned}
$$

Since $\xi=\xi(s)$ is a positive function, the identity (3.1) together with (3.2) above yields the required inequality (3.3.1). If $\ddot{\sigma}>0$ on $I_{X}$, then by

$$
\ddot{\sigma}(u)\left(\partial u_{\omega} \wedge \bar{\partial} u_{\omega}, \partial f \wedge \bar{\partial} f\right)_{\omega} \geq C(\partial \psi \wedge \bar{\partial} \psi, \partial f \wedge \bar{\partial} f)_{\omega},
$$


we see from (3.3.6) that the required inequality (3.3.2) holds as follows:

$$
\begin{aligned}
(P-\partial / \partial t) \hat{F} \geq & -(d \hat{F}, d f)_{\omega}-t^{-1} \hat{F}+n^{-1} t\left\{t^{-1} \hat{F}-2^{-1}(d \psi, d f)_{\omega}\right\}^{2} \\
& +t \ddot{\sigma}(u)\left(\partial u_{\omega} \wedge \bar{\partial} u_{\omega}, \partial f \wedge \bar{\partial} f\right)_{\omega} \\
\geq & -(d \hat{F}, d f)_{\omega}-t^{-1} \hat{F}+n^{-1} t\left\{t^{-1} \hat{F}-2^{-1}(d \psi, d f)_{\omega}\right\}^{2} \\
& +t C(\partial \psi \wedge \bar{\partial} \psi, \partial f \wedge \bar{\partial} f)_{\omega} \\
\geq & -(d \hat{F}, d f)_{\omega}-t^{-1} \hat{F}+n^{-1} t\left\{t^{-1} \hat{F}-2^{-1}(d \psi, d f)_{\omega}\right\}^{2} \\
& +C n^{-1} t\left\{2^{-1}(d \psi, d f)_{\omega}\right\}^{2} \\
= & -(d \hat{F}, d f)_{\omega}-t^{-1} \hat{F}+n^{-1}(C+1)^{-1} C t^{-1} \hat{F}^{2} \\
& +n^{-1} t(C+1)\left\{(C+1)^{-1} t^{-1} \hat{F}-2^{-1}(d \psi, d f)_{\omega}\right\}^{2} \\
\geq & -(d \hat{F}, d f)_{\omega}-t^{-1} \hat{F}+C_{0} t^{-1} \hat{F}^{2} .
\end{aligned}
$$

Proposition 3.4. Let $v=v(x, t) \in \Sigma$ be a positive solution of the heat equation $(P-\partial / \partial t) v=0$ on $M \times(0, \infty)$. Put $v_{t}:=\partial v / \partial t$. Moreover, by setting $f:=\log v$, we define $\hat{F}:=t\left\{(\bar{\partial} f, \bar{\partial} f)_{\omega}-f_{t}\right\}=t\left\{v^{-2}(\bar{\partial} v, \bar{\partial} v)_{\omega}-v_{t} \cdot v^{-1}\right\}$. Assume that

$$
\lim _{t \rightarrow 0} \sup _{(0, t] \times M} \hat{F} \leq n
$$

Then we have the following:

(a) If (1.5) is the case, then $(\bar{\partial} v, \bar{\partial} v)_{\omega}-v_{t} \cdot v \leq C_{1}^{-1}\left(n t^{-1}+k\right) v^{2}$ on $M \times(0, \infty)$.

(b) If (1.4) is the case, then $(\bar{\partial} v, \bar{\partial} v)_{\omega}-v_{t} \cdot v \leq C_{0}^{-1} t^{-1} v^{2}$ on $M \times(0, \infty)$.

Proof. (a) By the definition above, $f$ satisfies the equation $(P-\partial / \partial t) f=$ $-(\bar{\partial} f, \bar{\partial} f)_{\omega}$ on $M \times(0, \infty)$. By (a) of Proposition 3.3, $F:=\xi(\psi) \hat{F}$ satisfies (3.3.1) above. Now for each positive real number $T>0$, we claim the inequality

$$
F \leq C_{1}^{-1}(n+k T) \quad \text { on } M \times(0, T] .
$$

Assume the contrary. Since, by (3.2), $C_{1}^{-1} \geq \xi(s)$ for all $s$, the above (3.4.1) implies $C_{1}^{-1}(n+$ $k T)>\lim _{t \rightarrow 0} \sup _{(0, t] \times M} F$. Hence, for some $T>0$, the function $F$ takes a maximum at $\left(x_{0}, t_{0}\right)$ on $M \times(0, T]$ satisfying

$$
F\left(x_{0}, t_{0}\right)>C_{1}^{-1}(n+k T)>0 .
$$

Then by the maximality, $P F\left(x_{0}, t_{0}\right) \leq 0, d F_{\mid\left(x_{0}, t_{0}\right)}=0$, and $(\partial F / \partial t)\left(x_{0}, t_{0}\right) \geq 0$. By (1.5) and (3.2), the inequality (3.3.1) yields

$$
0 \geq-\frac{k F\left(x_{0}, t_{0}\right)}{n}-\frac{F\left(x_{0}, t_{0}\right)}{t_{0}}+C_{1} \frac{F\left(x_{0}, t_{0}\right)^{2}}{n t_{0}},
$$

which would imply that $F\left(x_{0}, t_{0}\right) \leq C_{1}^{-1}\left(n+k t_{0}\right) \leq C_{1}^{-1}(n+k T)$ in contradiction. Thus, we obtain (3.4.2) for all $T>0$. Hence,

$$
F \leq C_{1}^{-1}(n+k t) \quad \text { on } M \times(0, \infty) .
$$


Since $\xi(s) \geq 1$ for all $s$, it follows that $\hat{F} \leq C_{1}^{-1}(n+k t)$ on $M \times[0, \infty)$, and by multiplying both sides by $t^{-1} v^{2}$, we obtain the required inequality.

(b) If (1.4) is the case, then by (3.4.1) and the inequality $C_{0}^{-1}>n$, the maximal principle as above and (3.3.2) imply

$$
\hat{F} \leq C_{0}^{-1} \quad \text { on } M \times(0, T]
$$

for all positive real numbers $T>0$. By multiplying both sides of the above inequality by $t^{-1} v^{2}$, we obtain the required inequality.

For any $x, y \in M$, we choose a distance-minimizing geodesic $\gamma:[0,1] \rightarrow \boldsymbol{R}, s \mapsto \gamma(s)$, connecting $y=\gamma(0)$ and $x=\gamma(1)$. Write $(\dot{\gamma}, \dot{\gamma})_{\omega}$ simply as $\|\dot{\gamma}\|_{\omega}^{2}$, where $\dot{\gamma}:=\gamma_{*}(\partial / \partial t)$. Then the distance $r(x, y)$ of the points $x, y$ on the Kähler manifold $(M, \omega)$ is given by

$$
r(x, y)^{2}=\int_{0}^{1}\|\dot{\gamma}\|_{\omega}^{2} d s .
$$

Proposition 3.5. Let $v=v(x, t) \in \Sigma$ be a positive solution on $M \times(0, \infty)$ of the equation $(P-\partial / \partial t) v=0$. Assume that

$$
\lim _{t \rightarrow 0} \sup _{(0, t] \times M} t\left\{v^{-2}(\bar{\partial} v, \bar{\partial} v)_{\omega}-v_{t} \cdot v^{-1}\right\} \leq n,
$$

where $v_{t}:=\partial v / \partial t$. Then the following inequality holds for all $\left(x, t_{1}\right),\left(y, t_{2}\right) \in M \times(0, \infty)$ with $0<t_{1}<t_{2}$ :

(a) If (1.5) is the case, then

$$
v\left(x, t_{1}\right) \leq v\left(y, t_{2}\right)\left(t_{2} / t_{1}\right)^{n / C_{1}} \exp \left\{\left(t_{2}-t_{1}\right)^{-1} r(x, y)^{2} / 2+C_{1}^{-1} k\left(t_{2}-t_{1}\right)\right\} .
$$

(b) If (1.4) is the case, then

$$
v\left(x, t_{1}\right) \leq v\left(y, t_{2}\right)\left(t_{2} / t_{1}\right)^{1 / C_{0}} \exp \left\{\left(t_{2}-t_{1}\right)^{-1} r(x, y)^{2} / 2\right\} .
$$

Proof. (a) For $\gamma$ as above, let $\tilde{\gamma}:[0,1] \rightarrow M \times(0, T]$ be the path defined by $\tilde{\gamma}(s):=\left(\gamma(s),(1-s) t_{2}+s t_{1}\right)$. In view of $\tilde{\gamma}(0):=\left(y, t_{2}\right)$ and $\tilde{\gamma}(1):=\left(x, t_{1}\right)$, we obtain

$$
\begin{aligned}
& \log \left\{\frac{v\left(x, t_{1}\right)}{v\left(y, t_{2}\right)}\right\}=\log \{v \circ \tilde{\gamma}(1)\}-\log \{v \circ \tilde{\gamma}(0)\}=\int_{0}^{1}\left(\frac{d}{d s} \log v \circ \tilde{\gamma}\right) d s \\
& \quad=\int_{0}^{1} \tilde{\gamma}^{*}\left\{\left\langle v^{-1} d v, \dot{\gamma}\right\rangle-\left(t_{2}-t_{1}\right) v^{-1} v_{t}\right\} d s \\
& \leq \int_{0}^{1} \tilde{\gamma}^{*}\left\{\left\langle v^{-1} d v, \dot{\gamma}\right\rangle+\left(t_{2}-t_{1}\right) C_{1}^{-1}\left(n t^{-1}+k\right)-\left(t_{2}-t_{1}\right) v^{-2}(\bar{\partial} v, \bar{\partial} v)_{\omega}\right\} d s,
\end{aligned}
$$

where in the last inequality, $t=(1-s) t_{2}+s t_{1}$, and (a) of Proposition 3.4 is applied. On the other hand, by the Cauchy-Schwarz inequality, we have

$$
\left\langle v^{-1} d v, \dot{\gamma}\right\rangle \leq \sqrt{2} v^{-1}\left\{(\bar{\partial} v, \bar{\partial} v)_{\omega}\right\}^{1 / 2}\|\dot{\gamma}\| \leq\left\{\left(t_{2}-t_{1}\right)^{-1}\|\dot{\gamma}\|^{2} / 2\right\}+\left(t_{2}-t_{1}\right) v^{-2}(\bar{\partial} v, \bar{\partial} v)_{\omega} .
$$


All these together imply the required estimate:

$$
\begin{aligned}
\log \left\{\frac{v\left(x, t_{1}\right)}{v\left(y, t_{2}\right)}\right\} & \leq \int_{0}^{1}\left\{\frac{1}{2\left(t_{2}-t_{1}\right)}\|\dot{\gamma}\|^{2}+\frac{\left(t_{2}-t_{1}\right)}{C_{1}}\left(\frac{n}{(1-s) t_{2}+s t_{1}}+k\right)\right\} d s \\
& =\left\{\left(t_{2}-t_{1}\right)^{-1} r(x, y)^{2} / 2\right\}+\left(n / C_{1}\right) \log \left(t_{2} / t_{1}\right)+C_{1}^{-1} k\left(t_{2}-t_{1}\right) .
\end{aligned}
$$

(b) If (1.4) is the case, then we can apply (b) of Proposition 3.4. We here observe that the inequlity in (b) of Proposition 3.4 is obtained formally from the inequality (a) of Proposition 3.4 by replacing the pair $\left(C_{1}^{-1} n, C_{1}^{-1} k\right)$ by $\left(C_{0}^{-1}, 0\right)$. Then we obtain the required (3.5.3) from the inequality (3.5.2) by such a replacement.

4. Proof of Theorem A. In this section, we assume $\psi=\sigma\left(u_{\omega}\right)$ and $\operatorname{Ric}\left(h_{\psi / 2}\right) \geq 0$ as in the last section. In view of (2.1), let $0=\mu_{0}<\mu_{1} \leq \cdots \leq \mu_{i} \leq \ldots$ be the increasing sequence of the eigenvalues of the operator $-P$, and let $\mathcal{E}:=\left\{f_{0}, f_{1}, \ldots, f_{i}, \ldots\right\}$ be the corresponding set of eigenfunctions in $C^{\infty}(M)_{R}$ which are orthonormal, i.e.,

$$
\left\langle\left\langle f_{i}, f_{j}\right\rangle\right\rangle=\delta_{i j}
$$

for all $i$ and $j$. Then $f_{0}$ is nothing but the constant function $\operatorname{vol}_{\psi}(M)^{-1 / 2}$ on $M$. In view of (2.1), the fundamental solution $H=H(x, y, t)$ in the introduction is expressible as

$$
H(x, y, t)=\sum_{i=0}^{\infty} e^{-\mu_{i} t} f_{i}(x) f_{i}(y) .
$$

For $\tilde{H}(x, y, t)$ as in the introduction, by translating the result in [LY; Lemma 3.2] word for word to our situation, we immediately obtain:

FACT 4.1. Let $Z_{1}, Z_{2}$ be measurable subsets of $M$ which are preserved by the $Q$ action on $M$. Let $T>0, \delta>0, \tau>0$ be real numbers such that $\tau<(1+2 \delta) T$. For each $x \in M$, define $F_{x, T}(y, t)$ to be the integrals

$$
\int_{Z_{1}} H(y, z, t) \tilde{H}(x, z, T) h_{\psi}^{n}(z)=\int_{Z_{1}} \tilde{H}(y, z, t) \tilde{H}(x, z, T) h_{\psi}^{n}(z)
$$

for all $y \in M$ and $0<t \leq \tau$. Put $\underline{r}\left(x, Z_{i}\right):=\inf _{z \in Z_{i}} r(x, z)$ and $\bar{r}\left(x, Z_{i}\right):=\sup _{z \in Z_{i}} r(x, z)$ for each $i \in\{1,2\}$. Then

$$
\int_{Z_{2}}\left\{F_{x, T}(z, \tau)\right\}^{2} h_{\psi}^{n}(z) \leq \exp \left\{\frac{-\underline{r}\left(x, Z_{1}\right)^{2}}{(1+2 \delta) T}+\frac{\bar{r}\left(x, Z_{2}\right)^{2}}{(1+2 \delta) T-\tau}\right\} F_{x, T}(x, T) .
$$

In view of (2.1), since the principal parts of the operators $P$ and $\square_{\omega}$ coincide, we can describe the asymptotic behavoiur of $H(x, y, t)$ by the following identity:

$$
H(x, y, t)=(4 \pi t)^{-n} \exp \left\{-t^{-1} r(x, y)^{2} / 2+\phi(x, y, \sqrt{t})\right\} \quad \text { on } M \times M \times(0, \infty),
$$

where $\phi \in C^{\infty}(M \times M \times[0, \infty))_{\boldsymbol{R}}$. For the exterior differentiation $d_{y}=\partial_{y}+\bar{\partial}_{y}$ on the second factor $M$ of $M \times M \times[0, \infty)$, the function $\rho:=-t^{-1} r(x, y)^{2} / 2$ satisfies $\left(\bar{\partial}_{y} \rho, \bar{\partial}_{y} \rho\right)_{\omega}=$ 
$2^{-1}\left(d_{y} \rho, d_{y} \rho\right)_{\omega}=\partial \rho / \partial t$ (cf. [LY]). Hence, for each fixed $x \in M$,

$\lim _{t_{0} \rightarrow 0} \sup _{\left(0, t_{0}\right] \times M} t\left\{H(x, y, t)^{-2}\left(\bar{\partial}_{y} H(x, y, t), \bar{\partial}_{y} H(x, y, t)\right)_{\omega}-H(x, y, t)^{-1} \frac{\partial}{\partial t} H(x, y, t)\right\} \leq n$,

where the supremum is taken over all $(t, y)$ in $\left(0, t_{0}\right] \times M$. Similar inequality holds also for the average $\tilde{H}(x, y, t)$ of $H(x, y, t)$ by the $Q$-action, because $Q$ acts isometrically on the Kähler manifold $(M, \omega)$. By this together with Propositions 3.5 and Fact 4.1, we can now prove Theorem A.

(4.2) Proof of Theorem A. (a) Assume that (1.5) holds. Let $\tau=(1+\delta) T$ in Fact 4.1 , and consider the function $F_{x, T}(y, t)$, where $Z_{1}:=\tilde{B}_{y}(\sqrt{t})$ and $Z_{2}:=\tilde{B}_{x}(\sqrt{t})$. Then for all $x, y \in M$, applying Proposition 3.5 to the function $F_{x, T}(y, t)$ with $\left(t_{1}, t_{2}\right)=(T, \tau)$, we obtain

$$
F_{x, T}(x, T) \leq F_{x, T}(y, \tau)(1+\delta)^{n / C_{1}} \exp \left\{T^{-1} \delta^{-1} r(x, y)^{2} / 2+C_{1}^{-1} k T \delta\right\} .
$$

Integrating the square of this over all $y$ in $Z_{2}=\tilde{B}_{x}(\sqrt{t})$, we obtain

$$
\begin{aligned}
\operatorname{vol}_{\psi}\left(\tilde{B}_{x}(\sqrt{t})\right) F_{x, T}(x, T)^{2} \\
\quad \leq\left\{\int_{Z_{2}} F_{x, T}(y, \tau)^{2} h_{\psi}^{n}(y)\right\}(1+\delta)^{2 n / C_{1}} \exp \left\{T^{-1} \delta^{-1} \underline{t}+2 C_{1}^{-1} k T \delta\right\} \\
\leq(1+\delta)^{2 n / C_{1}} \exp \left\{\frac{\underline{-r}\left(x, Z_{1}\right)^{2}}{(1+2 \delta) T}+\frac{2 \underline{t}}{T \delta}+2 C_{1}^{-1} k T \delta\right\} F_{x, T}(x, T),
\end{aligned}
$$

where in the last inequality, we use (4.1.2) and the inequality $\underline{t} \geq \bar{r}\left(x, Z_{2}\right)^{2}$ together with $\tau=(1+\delta) T$. Since $F_{x, T}(x, T)=\int_{Z_{1}} \tilde{H}(x, z, T)^{2} h_{\psi}^{n}(z)>0$, dividing $(4.2 .1)$ by $F_{x, T}(x, T)$, we obtain

$$
\begin{aligned}
\operatorname{vol}_{\psi}\left(\tilde{B}_{x}(\sqrt{t})\right) \int_{Z_{1}} \tilde{H}(x, z, T)^{2} h_{\psi}^{n}(z) \\
\quad \leq(1+\delta)^{2 n / C_{1}} \exp \left\{\frac{-\underline{r}\left(x, Z_{1}\right)^{2}}{(1+2 \delta) T}+\frac{2 \underline{t}}{T \delta}+2 C_{1}^{-1} k T \delta\right\} .
\end{aligned}
$$

Apply (3.5.2) of Proposition 3.5 to the function $\tilde{H}(x, z, T)$ in $z$ with $\left(t_{1}, t_{2}\right)=(t, T)$, where we set $T=(1+\delta) t$. Then for all $x, y, z \in M$,

$$
\tilde{H}(x, y, t)^{2} \leq(1+\delta)^{2 n / C_{1}} \tilde{H}(x, z, T)^{2} \exp \left\{\frac{r(y, z)^{2}}{t \delta}+2 C_{1}^{-1} k t \delta\right\} .
$$

Integrate this over all $z$ in $Z_{1}=\tilde{B}_{y}(\sqrt{t})$ and then multiply it by $\operatorname{vol}_{\psi}\left(\tilde{B}_{x}(\sqrt{t})\right)$. Then, by (4.2.2), we see that $\operatorname{vol}_{\psi}\left(\tilde{B}_{x}(\sqrt{t})\right) \operatorname{vol}_{\psi}\left(\tilde{B}_{y}(\sqrt{t})\right) \tilde{H}(x, y, t)^{2}$ is bounded from above by

$$
(1+\delta)^{4 n / C_{1}} \exp \left\{\frac{\bar{r}\left(y, Z_{1}\right)^{2}}{t \delta}+2 C_{1}^{-1} k t \delta\right\} \exp \left\{\frac{-\underline{r}\left(x, Z_{1}\right)^{2}}{(1+2 \delta) T}+\frac{2 \underline{t}}{T \delta}+2 C_{1}^{-1} k T \delta\right\} .
$$


Note that $\bar{r}\left(y, Z_{1}\right)^{2} \leq \underline{t}, \underline{r}\left(x, Z_{1}\right) \geq\{r(x, y)-\sqrt{t}\}_{+}$and $T=(1+\delta) t$. Hence,

$$
\operatorname{vol}_{\psi}\left(\tilde{B}_{x}(\sqrt{t})\right)^{1 / 2} \operatorname{vol}_{\psi}\left(\tilde{B}_{y}(\sqrt{t})\right)^{1 / 2} \tilde{H}(x, y, t)
$$

$$
\leq(1+\delta)^{2 n / C_{1}} \exp \left\{\frac{-\{r(x, y)-\sqrt{t}\}_{+}^{2}}{2 t\left(1+3 \delta+2 \delta^{2}\right)}+\frac{3+\delta}{2 \delta(1+\delta)} \cdot \stackrel{t}{=}+t \delta(2+\delta) C_{1}^{-1} k\right\} .
$$

Put $\varepsilon:=(3 \delta / 2)+\delta^{2}$ with $0 \leq \delta \leq 1 / 2$. Then $\delta$ is regarded as a function in $0<\varepsilon \leq 1$. For such $\varepsilon>0$, we see that $\delta \leq \sqrt{\varepsilon},\{2 \delta(1+\delta)\}^{-1}(3+\delta) \leq(7 / 3) \varepsilon^{-1}$, and $\delta(2+\delta) \leq 4 \varepsilon / 3$. Then the required estimate (1.7) follows immediately from (4.2.3).

(b) Next, assume that (1.4) holds. Then by Proposition 3.5, we have the statement (3.5.3) which is formally obtained from the statement (3.5.2) by replacing the pair $\left(n / C_{1}\right.$, $\left.C_{1}^{-1} k\right)$ by $\left(1 / C_{0}, 0\right)$. Hence, the arguments in showing (1.7) allow us to obtain the required inequality (1.8) by such replacements.

REMARK 4.3. (1) In (a) of Theorem A, applying (1.7) to the case $\varepsilon=1$ and $t=$ $\operatorname{Diam}(M, \omega)^{2}$, we obtain the following estimate of $\tilde{H}$ for all $x \in M$ :

$$
\tilde{H}\left(x, x, \operatorname{Diam}(M, \omega)^{2}\right) \leq 4^{n / C_{1}} \operatorname{vol}_{\psi}(M)^{-1} \exp \left\{\frac{7}{3}+\frac{4}{3} C_{1}^{-1} k \operatorname{Diam}(M, \omega)^{2}\right\} .
$$

(2) In (b) of Theorem A, applying (1.8) to the case $\varepsilon=1$ and $t=\operatorname{diam}(M, \omega)^{2}$, we obtain the following estimate of $\tilde{H}$ for all $x \in M$ :

$$
\tilde{H}\left(x, x, \operatorname{Diam}(M, \omega)^{2}\right) \leq 4^{1 / C_{0}} e^{7 / 3} \operatorname{vol}_{\psi}(M)^{-1} .
$$

5. Proof of Theorem B. Consider the space $C^{\infty}(M)_{\boldsymbol{R}}^{\text {inv }}:=\left\{f \in C^{\infty}(M)_{\boldsymbol{R}} ; X_{\boldsymbol{R}} f=\right.$ $0\}$ of all $Q$-invariant smooth functions on $M$. Let $0=\tilde{\mu}_{0}<\tilde{\mu}_{1} \leq \cdots \leq \tilde{\mu}_{i} \leq \ldots$ be the increasing sequence of the eigenvalues of the operator $-P$ on $C^{\infty}(M)_{\boldsymbol{R}}^{\text {inv }}$, and let $\tilde{\mathcal{E}}:=\left\{\tilde{f}_{0}, \tilde{f}_{1}, \ldots, \tilde{f}_{i}, \ldots\right\}$ be the corresponding orthonormal eigenfunctions in $C^{\infty}(M)_{\boldsymbol{R}}^{\text {inv }}$. Since every eigenspace of the operator $-P$ on $C^{\infty}(M)_{R}$ is preserved by the $Q$-action, we may assume that $\tilde{\mathcal{E}}$ above is a subset of $\mathcal{E}$ in the last section. Then on $M \times M \times(0, \infty)$, we write

$$
\tilde{H}(x, y, t)=\sum_{i=0}^{\infty} e^{-\tilde{\mu}_{i} t} \tilde{f}_{i}(x) \tilde{f}_{i}(y) .
$$

Note that $\tilde{\mu}_{1} \geq \lambda_{1}(\omega, \psi)$ by $D_{\psi} \tilde{f}_{1}=\bar{D}_{\psi} \tilde{f}_{1}=P \tilde{f}_{1}=\tilde{\mu}_{1} \tilde{f}_{1}$. Put $H_{0}(x, y, t):=H(x, y, t)-$ $\operatorname{vol}_{\psi}(M)^{-1}$ and $\tilde{H}_{0}(x, y, t):=\tilde{H}(x, y, t)-\operatorname{vol}_{\psi}(M)^{-1}$. Since $G_{\omega}(x, y)=\int_{0}^{\infty} H_{0}(x, y, t) d t$, we have

$$
\tilde{G}_{\omega}(x, y):=\int_{0}^{\infty} \tilde{H}_{0}(x, y, t) d t=\sum_{i=1}^{\infty} \tilde{\mu}_{i}^{-1} \tilde{f}_{i}(x) \tilde{f}_{i}(y) .
$$

Assume (1.6) as in the last two sections. Let $v>0$ be a positive real number, and on $M$, we assume $\operatorname{Ric}\left(h_{\omega}^{\psi / 2}\right) \geq v \omega$. Then by [M1; (1.7)], we have

$$
\operatorname{Diam}(M, \omega) \leq C_{2} / \sqrt{v},
$$


where we set $C_{2}:=2 \pi(2 n-1+4 c)^{1 / 2}$ for the positive real constant $c$ in Section 3. We now give a proof of Theorem $B$.

(5.4) Proof of Theorem B. As in Section 2, let $\lambda_{1}=\lambda_{1}(\omega, \psi)$ be the first positive eigenvalue of the operator $-D_{\psi}$. Since $\operatorname{Ric}\left(h_{\omega}^{\psi / 2}\right) \geq v \omega$ and $\lambda_{1}=\lambda_{1}(\omega, 2(\psi / 2))$, an application of Proposition 2.6 to $l=2$ now yields

$$
\tilde{\mu}_{1} \geq \lambda_{1} \geq v e^{-2 c} .
$$

Put $t_{0}:=\operatorname{Diam}(M, \omega)^{2}$ for simplicity. Since $\tilde{H}_{0}\left(x, x, t+t_{0}\right) \leq e^{-\tilde{\mu}_{1} t} \tilde{H}\left(x, x, t_{0}\right)$ for all $t \geq 0$, the inequalities (4.3.1) and (4.3.2) imply the following for all $t>0$ and $x \in M$ :

$$
\begin{cases}\tilde{H}_{0}\left(x, x, t_{0}+t\right) \leq e^{-\tilde{\mu}_{1} t} B_{1} & \text { if }(1.5) \text { is the case } \\ \tilde{H}_{0}\left(x, x, t_{0}+t\right) \leq e^{-\tilde{\mu}_{1} t} B_{2} & \text { if }(1.4) \text { is the case }\end{cases}
$$

where $B_{1}:=4^{n / C_{1}} \operatorname{vol}_{\psi}(M)^{-1} \exp \left\{(7 / 3)+(4 / 3) C_{1}^{-1} k t_{0}\right\}$ and $B_{2}:=4^{1 / C_{0}} e^{7 / 3} \operatorname{vol}_{\psi}(M)^{-1}$. Then by (5.1), we have $\left|\tilde{H}_{0}\left(x, y, t_{0}+t\right)\right| \leq \tilde{H}_{0}\left(x, x, t_{0}+t\right)^{1 / 2} \tilde{H}_{0}\left(y, y, t_{0}+t\right)^{1 / 2}$, and hence

$$
\left|\tilde{H}_{0}\left(x, y, t_{0}+t\right)\right| \leq e^{-\tilde{\mu}_{1} t} B_{\alpha},
$$

where $\alpha=1$ or 2 according as (1.5) or (1.4) holds. Therefore, by (5.2), it follows that

$$
\begin{aligned}
\tilde{G}_{\omega}(x, y) & \geq-\int_{0}^{t_{0}} \operatorname{vol}_{\psi}(M)^{-1} d t-\int_{t_{0}}^{\infty} e^{-\tilde{\mu}_{1}\left(t-t_{0}\right)} B_{\alpha} d t \geq-t_{0} \operatorname{vol}_{\psi}(M)^{-1}-\tilde{\mu}_{1}^{-1} B_{\alpha} \\
& \geq-v^{-1}\left\{\operatorname{vol}_{\psi}(M)^{-1} C_{2}^{2}+e^{2 c} B_{\alpha}\right\}
\end{aligned}
$$

where in the last inequality, we used (5.3) and (5.4.1). Thus, if (1.5) is the case, then the following inequality holds for all $x, y \in M$ with $x \neq y$ :

$$
\begin{aligned}
\tilde{G}_{\omega}(x, y) & \geq-v^{-1}\left\{\operatorname{vol}_{\psi}(M)^{-1} C_{2}^{2}+e^{2 c} B_{1}\right\} \\
& =-\left\{v \operatorname{vol}_{\psi}(M)\right\}^{-1}\left\{C_{2}^{2}+e^{2 c} 4^{n / C_{1}} \exp \left(\frac{7}{3}+\frac{4}{3} C_{1}^{-1} k \operatorname{Diam}(M, \omega)^{2}\right)\right\} \\
& \geq-\left\{v \operatorname{vol}_{\psi}(M)\right\}^{-1}\left\{C_{2}^{2}+e^{2 c+(7 / 3)} 4^{n / C_{1}} \exp \left(\frac{4}{3} C_{1}^{-1} k \frac{C_{2}{ }^{2}}{v}\right)\right\} .
\end{aligned}
$$

Then for (a) of Theorem B, we obtain the required inequality by setting $k_{1}:=C_{2}{ }^{2}, k_{2}:=$ $e^{2 c+(7 / 3)} 4^{n / C_{1}}$ and $k_{3}:=(4 / 3) C_{1}^{-1} C_{2}{ }^{2}$. Next, if (1.4) is the case, then we have

$$
\begin{aligned}
\tilde{G}_{\omega}(x, y) & \geq-v^{-1}\left\{\operatorname{vol}_{\psi}(M)^{-1} C_{2}^{2}+e^{2 c} B_{2}\right\} \\
& =-\left\{v \operatorname{vol}_{\psi}(M)\right\}^{-1}\left(C_{2}^{2}+e^{2 c+(7 / 3)} 4^{1 / C_{0}}\right),
\end{aligned}
$$

and for (b) of Theorem B also, the required inequality follows immediately with $k_{0}:=C_{2}{ }^{2}+$ $e^{2 c+(7 / 3)} 4^{1 / C_{0}}$.

REMARK 5.5. The quantity $\operatorname{vol}_{\psi}(M)=\int_{M} e^{-\psi} \omega^{n}$ satisfies $V e^{-\bar{c}} \leq \operatorname{vol}_{\psi}(M) \leq$ $V e^{-\underline{c}}$ for $V:=\int_{M} \omega^{n}$, where the real constants $\underline{c}, \bar{c}$ are as in Section 3. Hence, under the assumption of (1.6), we easily see that for a fixed $\sigma$ and a fixed Kähler class, the quantity $\operatorname{vol}_{\psi}(M)$ does not depend on the choice of $\omega$ in the Kähler class (see [M1]). 
REMARK 5.6. By (5.2), we have

$$
\left\{\begin{array}{l}
\int_{M} \tilde{G}(x, y) h_{\psi}^{n}(y)=0 \\
f(x)=\operatorname{vol}_{\psi}(M)^{-1} \int_{M} f(y) h_{\psi}^{n}(y)-\int_{M} \tilde{G}(x, y)(P f)(y) h_{\psi}^{n}(y),
\end{array}\right.
$$

for all $x \in M$ and all $f \in C^{\infty}(M)_{R}^{\text {inv }}$.

6. Proof of Theorem C. Let $\omega \in \mathcal{F}_{\sigma}$. Then for some $t \in[0,1], \omega$ satisfies the equation (1.9). On the other hand, there exists a function $\varphi \in C^{\infty}(M)_{\boldsymbol{R}}^{\text {inv }}$ such that $\omega=$ $\omega_{0}+\sqrt{-1} \partial \bar{\partial} \varphi$. Put $f_{\omega}:=-\sigma\left(u_{\omega}\right)-(1-t) \varphi$. Then by (1.1), (1.6) and (1.9),

$$
\begin{aligned}
\operatorname{Ric}(\omega) & =-\sqrt{-1} \partial \bar{\partial} \sigma\left(u_{\omega}\right)+\operatorname{Ric}\left(h_{\sigma\left(u_{\omega}\right)}\right)=-\sqrt{-1} \partial \bar{\partial} \sigma\left(u_{\omega}\right)+t \omega+(1-t) \omega_{0} \\
& =-\sqrt{-1} \partial \bar{\partial} \sigma\left(u_{\omega}\right)+t \omega+(1-t)(\omega-\sqrt{-1} \partial \bar{\partial} \varphi)=\omega+\sqrt{-1} \partial \bar{\partial} f_{\omega}
\end{aligned}
$$

on $M$. On the other hand, there exists a function $v_{\omega} \in C^{\infty}(M)_{\boldsymbol{R}}$ such that $X=\operatorname{grad}_{\omega}^{C} v_{\omega}$ and that $v_{\omega}$ satisfies the equation (see $[\mathrm{F} 1 ; \mathrm{p} .41]$ )

$$
\square_{\omega} v_{\omega}+\sqrt{-1} X f_{\omega}=-v_{\omega}
$$

Now by $X=\operatorname{grad}_{\omega}^{C} v_{\omega}$, we have $v_{\omega}=u_{\omega}+C_{3}$ for some real constant $C_{3}$. This constant is characterized as Futaki's invariant $F(X)$ of the vector field $X$ (see [F1; p. 54]) by integrating both sides of (6.1) over $M$ as follows:

$$
F(X):=\frac{1}{\sqrt{-1}} \int_{M}\left(X f_{\omega}\right) \frac{\omega^{n}}{V}=\int_{M} v_{\omega} \frac{\omega^{n}}{V}=\frac{1}{V} \int_{M} u_{\omega} \omega^{n}+C_{3}=C_{3},
$$

where $V:=\int_{M} \omega^{n}$. By combining (6.1) and (6.2), we obtain

$$
\begin{aligned}
\square_{\omega} u_{\omega} & =-u_{\omega}-C_{3}-\sqrt{-1} X f_{\omega} \\
& =-u_{\omega}-F(X)+\sqrt{-1} X\left\{\sigma\left(u_{\omega}\right)\right\}+\sqrt{-1}(1-t) X \varphi \\
& =-F(X)+\dot{\sigma}\left(u_{\omega}\right)\left(\bar{\partial} u_{\omega}, \bar{\partial} u_{\omega}\right)_{\omega}-t u_{\omega}-(1-t)\left(u_{\omega}-\sqrt{-1} X \varphi\right) \\
& \leq-F(X)-t u_{\omega}-(1-t) u_{\omega_{0}},
\end{aligned}
$$

where in the last inequality, we used the inequality $\dot{\sigma} \leq 0$ on $I_{X}$ and the identity $u_{\omega_{0}}=$ $u_{\omega}-\sqrt{-1} X \varphi$ (cf. [FM]) on $M$. Note that $\min _{M} u_{\omega}=\min _{M} u_{\omega_{0}}=l_{0}$ (see Section 1). Then the above (6.3) implies $\square_{\omega} u_{\omega} \leq C_{4}:=\max \left\{0,-F(X)-l_{0}\right\}$. Since $\ddot{\sigma} \geq 0$ on $I_{X}$, we finally obtain

$$
\begin{aligned}
\square_{\omega}\left\{-\sigma\left(u_{\omega}\right)\right\} & =-\ddot{\sigma}\left(u_{\omega}\right)\left(\bar{\partial} u_{\omega}, \bar{\partial} u_{\omega}\right)_{\omega}-\dot{\sigma}\left(u_{\omega}\right) \square_{\omega} u_{\omega} \\
& \leq-\dot{\sigma}\left(u_{\omega}\right) \square_{\omega} u_{\omega} \leq C_{4} C_{5},
\end{aligned}
$$

where $C_{5}:=\max _{s \in I_{X}}\{-\dot{\sigma}(s)\}$. Since the right-hand side $C_{4} C_{5}$ is a constant independent of the choice of $\omega$ in $\mathcal{F}_{\sigma}$, the proof of Theorem $\mathrm{C}$ is now complete. 


\section{REFERENCES}

[A1] T. Aubin, Réduction du cas positif de l'équation de Monge-Ampère sur les variété Kählériennes compactes à la démonstration d'une inégualité, J. Funct. Anal. 57 (1984), 143-153.

[B1] J. P. Bourguignon et Al., Preuve de la conjecture de Calabi, Astérisque 58, Soc. Math. France, 1978.

[BM] S. BANDO AND T. MABUCHI, Uniqueness of Einstein Kähler metrics modulo connected group actions, Algebraic Geometry, Sendai, 1985, 11-40, Adv. Stud. in Pure Math. 10, Kinokuniya and North-Holland, Tokyo and Amsterdam, 1987.

[D1] E. B. DAVIES, Gaussian upper bounds for the heat kernels of some second-order operators on Riemannian manifolds, J. Funct. Anal. 80 (1988), 16-32.

[DS] J. D. Deuschel AND D. W. STROock, Hypercontractivity and spectral gap of symmetric diffusions with applications to the stochastic Ising models, J. Funct. Anal. 92 (1990), 30-48.

[F1] A. FUTAKI, Kähler-Einstein metrics and integral invariants, Lect. Notes in Math. 1314, Springer-Verlag, Heidelberg, 1988.

[K1] S. KовAYASHI, Transformation groups in differential geometry, Ergeb. Math. Grenzgeb. 70, Springer-Verlag, Heidelberg, 1972.

[KK] A. Kasue AND H. Kumura, Spectral convergence of Riemannian manifolds, Tôhoku Math. J. 46 (1994), $147-179$.

[L1] A. Lichnerowicz, Géométrie des groupes de transformations, Dunod, Paris, 1958.

[L2] A. LichnerowicZ, Variétés kählériennes et première classe de Chern, J. Differential Geom. 1 (1967), 195224.

[LY] P. LI AND S. T. YAU, On the parabolic kernel of the Schrödinger operator, Acta Math. 156 (1986), 153-201.

[M1] T. MABUCHI, Multiplier Hermitian structures on Kähler manifolds, submitted to Nagoya Math. J.

[Mat] Y. Matsushima, Holomorphic vector fields on compact Kähler manifolds, Conf. Board Math. Sci. Regional Conf. Ser. in Math. 7, Amer. Math. Soc., Providence, R. I., 1971.

[N1] Y. NAKAGAWA, An isoperimetric inequality for orbifolds, Osaka J. Math. 30 (1990), 733-739.

[S1] A. G. SETTI, Gaussian estimates for the heat of the weighted Laplacian and fractal measures, Canadian J. Math. 44 (1992), 1061-1078.

[S2] A. G. SETTI, Eigenvalue estimates for the Laplacian with lower order terms on a compact Riemannian manifolds, Differential geometry: Riemannian geometry (Los Angeles, CA, 1990), 521-527, Proc. Sympos. Pure Math. 54, Amer. Math. Soc., Providence, R. I., 1993.

[TZ] G. TIAn AND X. H. ZHU, Uniqueness of Kähler-Ricci solitons , Acta Math. 184 (2000), 271-305.

DEPARTMENT OF MATHEMATICS

OSAKA UNIVERSITY

TOYONAKA, OSAKA, 560-0043

JAPAN 